\title{
AGGREGATE SUBGRADIENT SMOOTHING METHODS FOR LARGE SCALE NONSMOOTH NONCONVEX OPTIMISATION AND APPLICATIONS
}

\author{
NARGIZ SULTANOVA \\ (Received 19 December 2014; first published online 16 March 2015)
}

2010 Mathematics subject classification: primary 90C06; secondary 65K05, 90C90.

Keywords and phrases: nonsmooth optimisation, large-scale problems, mathematical programming.

Nonsmooth optimisation problems are problems which deal with minimisation or maximisation of functions that are not necessarily differentiable. They arise frequently in many practical applications, for example in engineering, machine learning and economics. In addition, some smooth problems can be reformulated as nonsmooth optimisation problems with a simpler structure or a smaller dimension. Despite the fact that there exist many algorithms for solving nonsmooth optimisation problems, the field is still very much in development. Nonsmooth nonconvex optimisation, in particular, is far from being considered a mature branch of optimisation.

The two main classes of algorithms for nonsmooth problems are subgradient and bundle methods. Subgradient methods are the simplest, while bundle methods are considered to be the most efficient methods for solving nonsmooth optimisation problems. In addition to these methods, smoothing techniques have been shown to be efficient for solving nonsmooth optimisation problems with special structures.

In this thesis, we present two new methods for nonsmooth nonconvex optimisation: a subgradient method for nonsmooth nonconvex minimisation and a subgradient smoothing method. The former combines the ideas from subgradient and bundle methods and the latter makes use of smoothing techniques. The aim of the subgradient method for nonsmooth nonconvex minimisation is to maintain the bundling approach of bundle methods and the ease of implementation of subgradient methods, while reducing computational effort, to make the algorithms more suitable for large scale problems. The subgradient smoothing method is aimed at applying smoothing techniques for general nonsmooth and, in particular, nonconvex optimisation problems.

Thesis submitted to the University of Ballarat, Victoria, Australia in May 2013; degree approved on 8 October 2013; principal supervisor: Adil Bagirov, associate supervisors: David Yost and Andrew Barton. (C) 2015 Australian Mathematical Publishing Association Inc. 0004-9727/2015 \$16.00 
We study the convergence of the methods and evaluate their performance by comparing them with other nonsmooth optimisation methods via numerical experiments on a wide range of test problems from the literature. The subgradient method for nonsmooth nonconvex minimisation is applied to study pumping cost minimisation problems in water management. The conventional model for pumping cost minimisation problems involves a large number of binary variables which makes it difficult to find a solution by applying the deterministic optimisation method. We present a nonsmooth nonconvex model of this problem which allows us to eliminate the binary variables and describe an algorithm for its solution that involves the use of the subgradient method for nonsmooth nonconvex minimisation.

NARGIZ SULTANOVA, School of Applied and Biomedical Sciences, Faculty of Science and Technology, Federation University, Ballarat, Victoria 3353, Australia

e-mail: n.sultanova@federation.edu.au 